\title{
PROCESO ELECTRODIALITICO CONTINUO EN LA REDUCCION DEL CONTENIDO DE IONES METALICOS EN LOS DRENAJES ACIDOS DE MINA
}

\section{CONTINUOUS ELECTRODIALITIC PROCESS IN THE REDUCTION OF THE CONTENT OF METAL IONS IN THE ACID MINE DRAINAGE}

\author{
César Paúl Ortiz Jahn ${ }^{4}$ Manuel Guerreros Meza'
}

\begin{abstract}
RESUMEN
La investigación en la fase preliminar para determinar la capacidad osmótica de los recipientes cerámicos comunes, y para el efecto del análisis de capacidad de separación de iones por el efecto electrodialítico, se han empleado lecturas conductométricas con la finalidad de determinar el transporte de iones a la otra fase líquida separada por el medio poroso. Los resultados indican una posibilidad de determinar las factibilidades de cuantificación analítica de la movilización iónica, que va a ser usado en el trabajo final para el proceso investigado. Los drenajes ácidos de mina son producidos como consecuencia de la lixiviación de sulfuros, atribuido especialmente a la interacción de las piritas, el agua y el oxígeno, lo cual al producir iones férricos y de hidrógeno, disuelven a las otras especies sulfuradas de metales bases que pueden existir en los cuerpos mineralizados. El objetivo final de este trabajo es el de proponer una técnica electroquímica que permita mitigar la contaminación de las aguas del túnel Victoria con altos contenidos de iones metálicos.
\end{abstract}

Palabras clave: Electrodiálisis, drenaje ácido de mina, conductometría.

\begin{abstract}
The investigation in the preliminary phase to determine the osmotic capacity of the common ceramic recipients, and for the effect of the ions separation capacity analysis by the electrodialitic effect itself there is employee to conductometric readings with the purpose to determine the transportation of ions to the other phase liquid separated by the porous middle. The results indicate a possibility to determine the feasibilities of analytic quantification of the mobilization ionic, that goes to to be used in the final work for the investigated process. The acid mine drainage is produced as a result of sulfurs leaching, attributed especially to the interaction of the pyrites, the water and the oxygen, which to produce ferric ions and hydrogen, they dissolve to the other sulfur species of metals bases that can exist in the bodies mineralized. The final objective of this work is that of proposing an electrochemical technique that permit to mitigate the contamination of the water of the tunnel Victory with high contents of metallic ions.
\end{abstract}

Keywords: Electrodialysis, acid mine drainage, conductometrics.

\footnotetext{
${ }^{4}$ Universidad Nacional del Centro del Perú. Facultad de Ingeniería Metalúrgica y de Materiales.
} 


\section{INTRODUCCION}

La generación de drenajes ácidos como consecuencia de los sistemas de explotación minero - metalúrgicos, es un problema inherente al control y manejo del medio ambiente. Un caso crítico, y que pretende abordar la presente investigación, es el que se presenta en el Túnel Victoria ubicado en la cuenca del río Yauli. El agua de mina de este túnel se descarga directamente sin tratamiento en el río Yauli, constituyendo uno de los principales contribuyentes en la contaminación de las aguas del río Mantaro, conjuntamente con las aguas del túnel Kingsmill. Las aguas del túnel Victoria se caracterizan por su alta acidez, con $\mathrm{pH}$ promedio de 2,5 á 4 , y por sus altos contenidos de iones metálicos como $\mathrm{Fe}^{3+}, \mathrm{Zn}^{2+}, \mathrm{Mn}^{2+}, \mathrm{Cd}^{2+}$ y $\mathrm{Cu}^{2+}$ que sobrepasan grandemente los Límites Máximos Permisibles (LMP). El caudal que aporta este túnel es de $350 \mathrm{~L} / \mathrm{s}$. Frente a esta problemática anteriormente expuesta, nos planteamos la interrogante:

¿Será posible reducir el contenido de iones metálicos de los drenajes ácidos de mina (DAM) que se vierten actualmente al río Yauli, hasta lograr niveles que permitan una calidad de agua apta para soportar la vida subacuática, mediante un proceso electrodialítico continuo?

El objetivo principal del estudio fue diseñar un proceso continuo a nivel de planta piloto para la separación de las especies metálicas disueltas en los DAM que se vierten actualmente al río Yauli, hasta lograr niveles que permitan una calidad de agua apta para soportar la vida subacuática, mediante un proceso electrodialítico continuo. Los objetivos específicos: desarrollar un prototipo de membrana para el proceso electrodialítico; proponer un modelo matemático para la simulación del proceso electrodialítico para la separación de los iones metálicos presentes en los DAM; diseñar la celda electrodialítica y el flujo tecnológico para el proceso propuesto; y evaluar a nivel de laboratorio la efectividad de la tecnología y el proceso propuesto. El objetivo en esta etapa preliminar fue establecer y analizar los sistemas cerámicos como membranas electrodialíticas.

\section{MATERIAL Y METODOS}

Luego de realizado el análisis teórico preliminar se propuso un esquema de desarrollo experimental que permitió encontrar parámetros operativos para investigar con más detalle y confirmar o demostrar la hipótesis planteada.

Básicamente, la experimentación consistió el desarrollo de una propuesta tecnológica electroquímica para la aplicación de la electrodiálisis a soluciones ácidas conteniendo iones metálicos en solución, de tal forma que pueda separarse una concentración concentrada y otra diluida.

Las principales actividades que se han previsto son: pruebas preliminares batch con sistemas cerámicos porosos que incluyen: preparación del equipo, preparación de soluciones estándar, preparación de métodos de análisis químicos para soluciones, corridas de pruebas batch preliminares.

\section{Diseño Metodológico:}

El sistema que fue empleado en la parte experimental del proyecto consistió en: suministro de energía, celda electroquímica, membranas, electrodos, sistema de medición y cablería.

\section{Área de Estudio:}

El efluente en estudio fueron las soluciones sintéticas, similares a los originados en el problema.

\section{Población y Muestra:}

Para efectos del estudio se consideró el empleo de soluciones sintéticas y naturales.

Las soluciones sintéticas fueron preparadas con agua destilada y reactivos de calidad Q. P. El estudio abarcó los efectos de la aplicación de la electrodiálisis sobre la variación del $\mathrm{pH}$ y la concentración de iones metálicos como el $\mathrm{Fe}^{++}, \mathrm{Cu}^{++}$, $\mathrm{Zn}^{++}$y $\mathrm{Mn}^{++}$. Por tal razón se prepararon soluciones estandarizadas de concentración $1 \mathrm{M}$, a partir de sus sulfatos respectivos. Como electrolito soporte se empleó soluciones de $\mathrm{Na}_{2} \mathrm{SO}_{4}$. La acidez de las soluciones fue controlada con la adición de $\mathrm{H}_{2} \mathrm{SO}_{4}$.

Técnicas de procesamiento y análisis de datos:

Las pruebas se llevaron a cabo independientemente para cada ión metálico en las condiciones indicadas en cada diagrama; el experimento ha consistido en someter a un proceso de electrólisis, empleando una estructura porosa de cerámica, para separar fases de electrolitos; se han realizado las mediciones de variación de la conductividad en el lado anódico (solución cargada con iones metálicos) y en el lado catódico (inicialmente la solución solo contiene el electrolito soporte), para el efecto hemos trabajado con soluciones de sulfato cúprico y sulfato férrico con concentraciones iniciales de 300 mg/l, para el lado catódico y una concentración inicial de sulfato de sodio de 10 $\mathrm{mg} / \mathrm{l}$, los $\mathrm{pH}$ trabajados fueron 2,5 y 4,0 , regulados con ácido sulfúrico, a temperatura ambiente, voltaje aplicado fue de 15 voltios, las pruebas duraron 4 horas, las mediciones se realizaron cada 30 minutos.

\section{RESULTADOS}

Luego de la aplicación del procedimiento señalado para cada solución en forma independiente se 
obtuvieron los resultados que se muestran en las figuras siguientes:

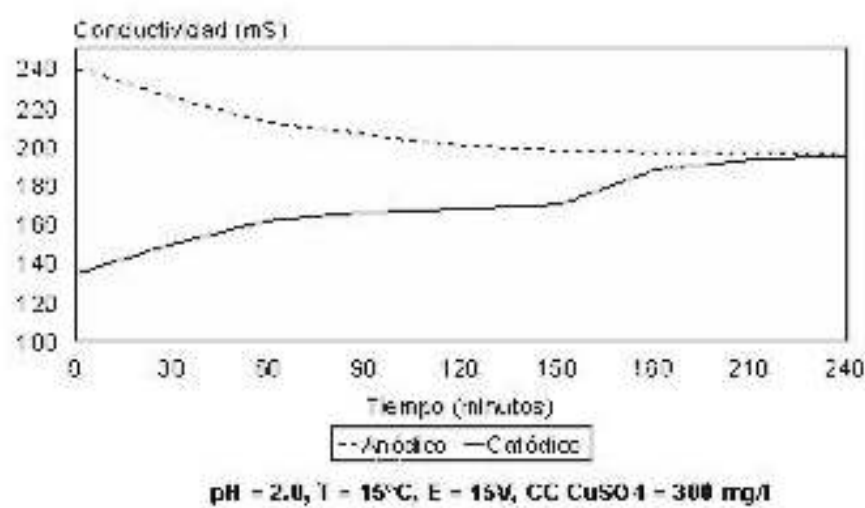

Figura 1. Comportamiento de la conductividad para soluciones de $\mathrm{CuSO}_{4}$.

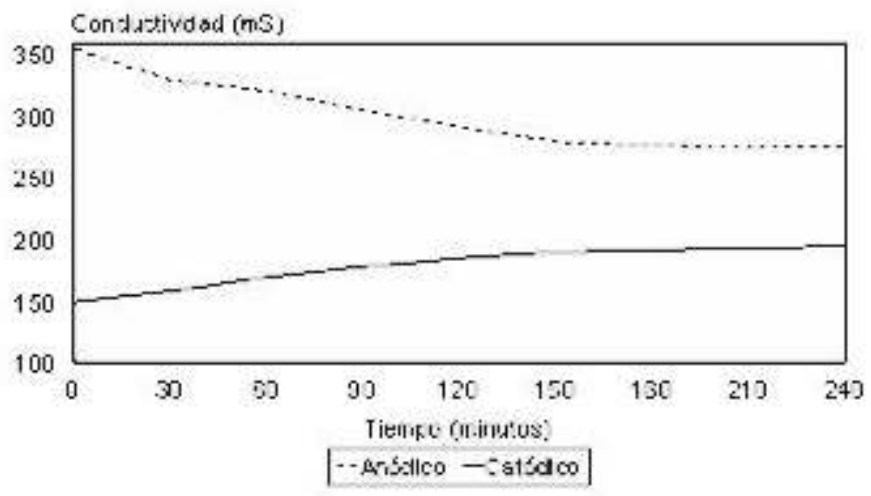

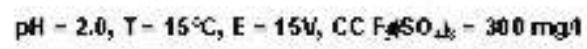

Figura 3. Comportaniento de la conductividad para soluciones de $\mathrm{Fe}_{2}\left(\mathrm{SO}_{4}\right)_{3}$.

\section{DISCUSIÓN}

El criterio de empleo de una barrera permeable, es decir el cerámico poroso surge de la metodología empleada por Treharne y Wright (1) dividieron la celda con una barrera de arena, en una propuesta para el tratamiento de drenajes ácidos de mina. Friederich y Knappnik (2) dividió la celda usando diafragmas cerámicos o membrana de intercambio iónico, y trataron DAM sintético conteniendo hierro (III). Los métodos conductométricos son empleados desde los años 1930 (I.M. Kolthoff(3))

En los resultados de las pruebas llevadas a cabo que se pueden observar en las figuras 1,2 y 3 , se puede indicar que la aplicación de la electrodiálisis en forma directa, sin la aplicación de membranas aniónicas o catiónicas, no es eficiente para la separación de iones metálicos cuando se emplea un

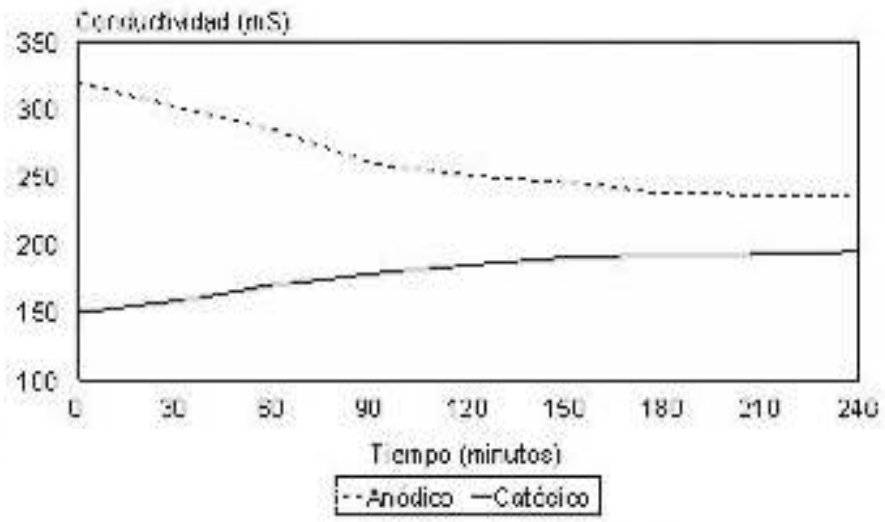

$\mathrm{pH}=4.0, \mathrm{I}=15^{\circ} \mathrm{C}, \mathrm{t}=15 \mathrm{v}, \mathrm{CC} \mathrm{CuS0} 4=300 \mathrm{mg} . \mathrm{I}$

Figura 2. Comportamiento de la conductividad para soluciones de $\mathrm{CuSO}_{4}$.

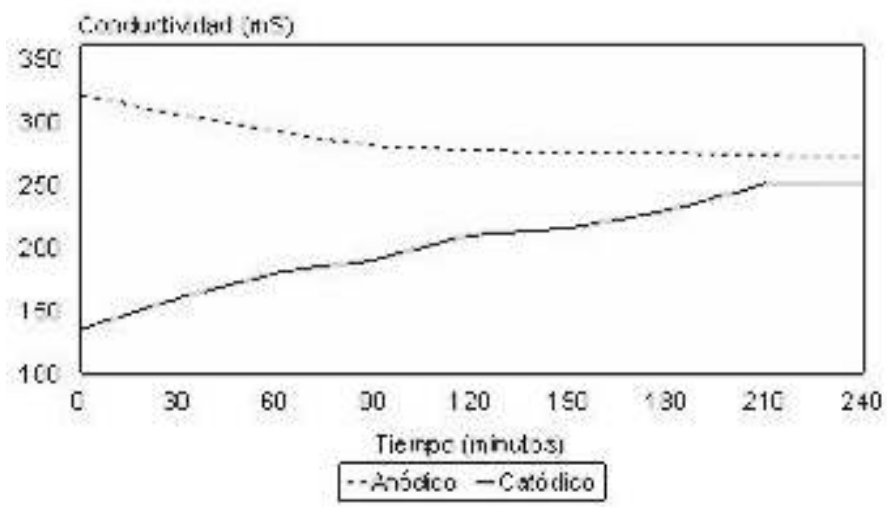

pH - 20, T $-15^{\circ} \mathrm{C}, \mathrm{E}-16 \mathrm{~V}, \mathrm{CC} \mathrm{F}_{4} \mathrm{SO}_{43}-300 \mathrm{mgh}$

Figura 4. Comportamiento de la conductividad para soluciones le $\mathrm{Fe}_{2}\left(\mathrm{SO}_{4}\right)_{3}$.

medio poroso cerámico. Esta conclusión se puede corroborar con lo indicado por Chartrand, M.M.G. y Bunce, N.J, (4).

Existe un mecanismo de transporte iónico identificable, en las fases aniónicas y catiónicas, ya que se observa una disminución gradual en la conductividad de las soluciones. El método conductométrico es aplicable para una medición indirecta de la concentración iónica de soluciones conteniendo iones metálicos. Es un principio fundamental de la titración conductométrica (I.M. Kolthoff(3)).

La aplicación de la electrodiálisis es una técnica muy acertada en el tratamiento de efluentes mineros por las particularidades que estas originan, considerando que es importante continuar la investigación propuesta empleando membranas aniónicas y catiónicas. 


\section{REFERENCIAS BIBLIOGRAFICAS}

1. Treharne RW, Wright DE. Acid mine water treatment process. US Patent $3823081 ; 1974$

2. Friedrich $\mathrm{HJ}$, Knappnik R. Method for raising the $\mathrm{pH}$ of acid waters, European Patent 0814060 A2; 1997.

3. 1. I.M. Kolthoff. Industrial \& Engineering Chemistry; 1930.

4. Chartrand MG, Bunce NJ. Electrochemical remediation of acid mine drainage. Journal of Applied Electrochemistry. 2003. 33: 259264.

Email: ceorja@yahoo.com 